\title{
The BC- MOS: New Bacterial Cellulose Bio-based MOS Gas Sensors
}

\author{
E. Núñez-Carmona ${ }^{1}$, A. Bertuna ${ }^{1}$, M. Abbatangelo ${ }^{1}$, V. Sberveglieri ${ }^{2,3}$, E. Comini ${ }^{1,3}$, G. Sberveglieri1,3 \\ ${ }^{1}$ Department of Information Engineering, University of Brescia, Via Branze 38, 25123, Brescia, Italy, \\ ${ }^{2}$ CNR-IBBR, Institute of Biosciences and Bioresources, Via Madonna del Piano 10, 50019, Sesto \\ Fiorentino (FI), Italy \\ ${ }^{3}$ NANO SENSOR SYSTEMS srl, Via Branze 38, 25123, Brescia, Italy. \\ e.nunezcarmona@unibs.it
}

\begin{abstract}
:
The possibility to create hybrid devices has arisen recently. A high interest has grown around the use of organic materials in engineering and electronic fields. One of the most interesting biomaterial is cellulose, which is the most abundant polymer in nature. In particular, it exhibits high degree of crystallinity, stability and withstanding to high temperatures, moreover may be easily tailored to its application requirements. $\mathrm{ZnO}$ has been used as active sensing material thanks to its well-known performances and to the room temperature sensing by UV light activation. A new generation of bacterial cellulose based MOX sensors have been successfully developed. The prototypes showed good response to ethanol, hydogren, nitrogen dioxide and acetone at room temperature, demonstrating the possibility to use cellulose as eco and environmental friendly transducers for, but not limited to, chemical sensors.
\end{abstract}

Key words: BC-MOX, Bacterial cellulose, MOX sensors, Zinc Oxide, thins films, photoactivation

\section{Introduction}

A high interest have been demonstrate in the engineering field regarding the use and creation of cellulose based hybrid devices due to the intrinsic properties of cellulose and its environmental friendly character. Bacterial cellulose (BC) it's a polymer it's formed by glucose molecules bounded by $\beta \rightarrow 1,4$ links [1]. It's produced by acetic acid bacteria from the Acetobacteraceae family, is composed by three-dimensional hierarchical structures presenting a very high degree of polymerization and crystallinity, which width is within $5-50 \mathrm{~nm}$. By controlling synthesis methods, the resulting microbial cellulose can be tailored to have specific properties. Principal Bacterial Cellulose features are: high purity, possibility to be functionalized by a broad range of reactions due to their reactive surface covered by $-\mathrm{OH}$ sites, high strength and good mechanical properties, high specific surface-area ratio, thermal stability and low thermal expansion coefficient.

Moreover, it's important to take into account the thermal stability of $\mathrm{BC}$ for their integration in real devices like smart substrates for chemical sensors, because the aforementioned processing technologies can easily get closer to $200^{\circ} \mathrm{C}$. The maximum processing and working temperature for composites integrating $\mathrm{BC}$ must be below $300^{\circ} \mathrm{C}$ as reported by thermos gravimetric analysis [1]. Currently the substrate used for the deposition of the sensing layer in MOXS sensors is made of alumina which fabrication is notoriously polluting [2]. However, $\mathrm{ZnO}$ as MOS sensing layers exhibit optimum performances as sensing material [3] and could be activated through the use of UV light avoiding the use energy to heat the system [4]. This work aims to the creation of a new generation BC-MOX sensor prepared using biomaterials as bacterial cellulose as a substrate (BCS) and $\mathrm{ZnO}$ as sensing layer.

\section{Materials \& Methods}

The microorganisms used to grow BCS in this study was Komagataeibacter sucrofermentans that were grown in 105 media and were incubated statically at $27^{\circ} \mathrm{C}$ for 10 days. Once harvested the membranes were purified by a soaking treatment in a solution of $2 \mathrm{M} \mathrm{NaOH}$ for $24 \mathrm{~h}$ at room temperature [1]. Subsequently membranes were rinsed until neutral $\mathrm{pH}$ was reached then exsiccated at $40^{\circ} \mathrm{C}$ during $24 \mathrm{~h}$ to obtain the BCS. The morphology of the as- 
prepared BCS was observed using a Zeiss LEO 1525 scanning electron microscope (SEM), operated at $1 \mathrm{keV}$. To build the sensor a deposition of a $\mathrm{ZnO}$ film was made over the BCS by means of DC magnetron sputtering, at room temperature with a pressure of $8 \mathrm{mbar}$ in O2 $50 \%$ and $\operatorname{Ar} 50 \%$ at 100 W RF (160 nm thickness). On the oxide surface interdigitated $\mathrm{Pt}$ contacts were deposited at room temperature by a DC magnetron sputtering as well. Finally the sensor was mounted on a TO case by gold wires. The response of the sensor to different kinds of chemical species was studied at room temperature in presence of UV light $(5 \mathrm{~mW} / \mathrm{mm} 2)$. The functional analysis of these devices was carried out using the flow through technique with ethanol and hydrogen, at different concentrations with a background of synthetic air.

\section{Results \& Discussion}

Figure $1 \mathrm{~A}$ (left) shows the arrangement of fibers at the surface of the BCS, the compact packing of the fibers runs evenly across the sample. Regarding the UV activation of metal oxides, it is fair to say that is promising method to achieve room-temperature sensitivity. Under UV light irradiation, $\mathrm{ZnO}$ currents increases about two orders of magnitude compared to

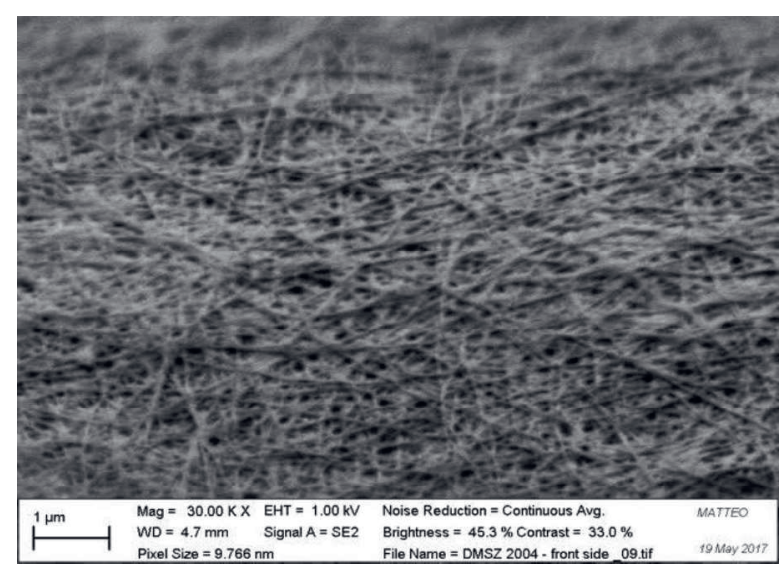

Fig.1.A) SEM image of the BCS surface,

\section{References}

[1] E. Fortunato, D. Gaspar, P. Duarte, L. Pereira, H. Águas, A. Vicente, F. Dourado, M. Gama, R. Martins, Optoelectronic Devices from Bacterial NanoCellulose Bacterial Nanocellulose, in book: M. Gama, F. Dourado, and S. Bielecki (Eds.), Bacterial Nanocellulose, From Biotechnology to Bio-Economy, 179-197 (2016).

[2] Z. Sun, F. Li, W. Bao, C. Wang, Mineral phase transition of desilicated high alumina fly ash with alumina extraction in mixed alkali solution International, Journal of Mineral Processing 153, 109-117(2016). dark values. In fact, $\mathrm{ZnO}$ films deposited on BCS showed a good response to hydrogen and ethanol at room temperature as UV light is used to enhance the chemical interactions between its surface and the environment, and the transduction into a variation of conductance. Figure $1 \mathrm{~B}$ (right) reports dynamic response of the sensor in the presence of UV light and dry air, in the presence of ethanol (pink) and $\mathrm{H} 2$ (yellow).

\section{Conclusions}

These results demonstrate the possibility to use these BSC as eco and environmental friendly substrates for, but not limited to, chemical sensors. The results show the future application of these BCS as chemical sensors thanks to their ambient friendly qualities, reduced costs, low thickness and the possibility to incorporate the sensing material. These prototypes can detect chemical species in environments where heating cannot be used to activate the sensing element. The quality of the response and the sensor itself may be optimized in term of performances modifying the cellulose features, to increase durability, and deposition methodologies. Furthermore, thanks to their thermal stability sensors can work at temperatures higher than room temperature.

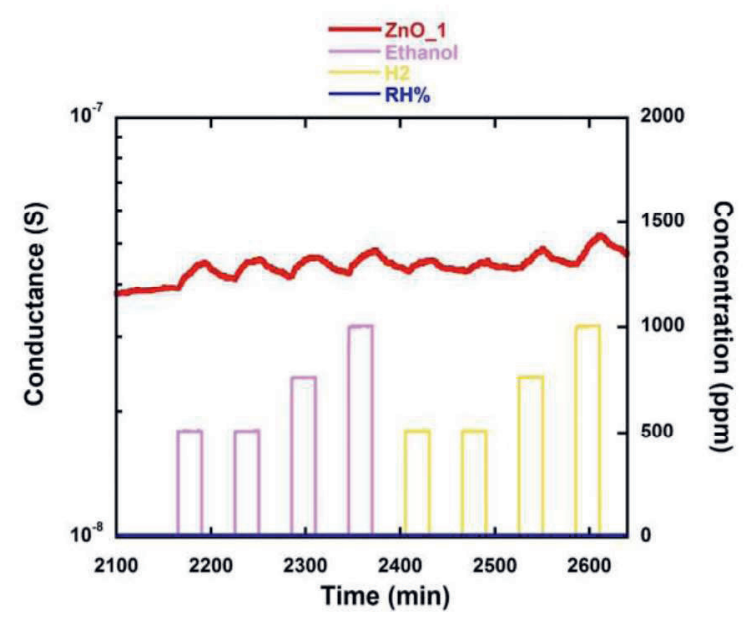

B) Sensor response to ethanol and hydrogen.

[3] G.Sberveglieri, I. Concina, E. Comini, M. Falasconi, M. Ferroni, V. Sberveglieri, Synthesis and integration of tin oxide nanowires into an electronic nose, Vacuum 86, 532-535 (2012).

[4] C. Baratto, S. Todros, G. Faglia, E. Comini, G.Sberveglieri, S. Lettieri, L. Santamaria, P. Maddalena, Luminescence response of $\mathrm{ZnO}$ nanowires to gas adsorption, Sensors and Actuators B: Chemical 140 (2), 461-466 (2009). 